\title{
The importance of the socioeconomic status of countries for mental disorders in old age: development of an epidemiological transition model
}

The prevalence of a disorder is a function of the incidence of the disorder and the duration of the illness (which may remit spontaneously, by treatment or death). The socioeconomic status of societies may influence the incidence and duration of mental illnesses in old age by influencing life expectancy and availability of appropriate healthcare services and treatments (Suh and Shah, 2001). The relationship between a proxy measure of the socioeconomic status (per capita gross national domestic product (GDP)) and (i) the life expectancy, (ii) child mortality rates and (iii) proxy measures of the quality and quantity of available healthcare services (the proportion of GDP spent on health, per capita expenditure on health and child mortality rates) was examined. The WHO website (www.who.int/countries/en/) provided data on these variables for 191 of the 192 countries for the year 2002. The inter-correlations between all the measured variables were examined using Pearson's correlation coefficient. Because of highly significant inter-correlations between all these variables, multiple linear regression analysis, with the Enter method, was undertaken with per capita GDP as the dependent variable, and all the variables that were significantly correlated with per capita GDP on univariate analysis as the independent variables.

Table S1 (available online as supplementary material attached to the electronic version of this letter at www.journals.cambridge.org/jid_IPG) illustrates the inter-correlations between all the measured variables. Multiple linear regression analysis, for males, revealed that the proportion of GDP spent on health $(\mathrm{P}<$ $0.00001)$ and per capita expenditure on health $(P<0.0001)$ were the only significant predictors of per capita GDP. The findings for females were identical.

This suggests that any influence of per capita GDP on the prevalence of mental disorder in old age may be mediated through the availability of appropriate health care services and treatments (as measured by the proxy markers of the proportion of GDP spent on health and per capita expenditure on health).

In socioeconomically less developed societies, various diseases (e.g. infectious diseases) associated with poor socioeconomic factors are common causes of death early in life due to paucity of appropriate healthcare services and treatments (Suh and Shah, 2001). This is supported by negative correlations between male and female child mortality rates and the proportion of GDP spent on health and per capita expenditure on health. This would lead to reduced life expectancy and is supported by positive correlations between male and female life expectancy

Email: ajit.shah@wlmht.nhs.uk 
and the proportion of GDP spent on health and per capita expenditure on health. Hence, the incidence (and therefore the prevalence) of a mental disorder that has an increased risk of development in old age will be low in these societies because fewer people will reach the age of increased risk, and this has been observed for dementia (Chandra et al., 2001; Suh and Shah, 2001). Moreover, in these societies, selective survival of those at reduced risk of developing mental disorders in old age due to genetic or constitutional factors may further compound this trend (Suh and Shah, 2001). In addition, those who do survive into old age may be at reduced risk of developing mental disorders because they may be able to tolerate additional hardship in old age better due to exposure to life-long adversity (Lindesay, 1981; Seiden, 1981) and this may reduce the incidence (and therefore the prevalence). This has been offered as an explanation for the low suicide rate among elderly African Americans and native Americans in the United States because they often have a lifelong history of socioeconomic deprivation (McIntosh, 1984). Such societies could be labeled as being at a low prevalence-low socioeconomic status stage in their development.

As the socioeconomic status of a given society improves, child mortality rates decline and life expectancy increases. This is supported by a negative and positive correlation between per capita GDP and child mortality rates and life expectancy respectively. This, in turn, will result in an increasing number of individuals reaching the age of increased risk of mental disorder in old age and an increase in its incidence (and therefore its prevalence). Also, as the socioeconomic status of societies improves, the availability of appropriate healthcare services and treatments will also improve - this is supported by positive correlations between per capita GDP and the proportion of GDP spent on health and per capita expenditure on health. This improved availability of appropriate healthcare services may, in turn, lead to increased life expectancy after the onset of the mental disorder in old age and a consequent increase in the prevalence. This will lead to a gradual societal transition from a low prevalence-low socioeconomic status stage to a high prevalence-low socioeconomic status stage.

As societies develop further they are likely to change from being socioeconomically less developed to being socioeconomically more developed. This will lead to further reduction in early mortality due to disorders associated with poor socioeconomic status, increased life expectancy, reduction in protective effects of adversity early in life and reduction in selective survival of those at reduced risk of suicide due to genetic or constitutional factors; this is supported by the negative and positive correlations between per capita GDP and child mortality rates and life expectancy respectively. The composite effect of these changes would be an increase in the number of individuals at increased risk of developing mental disorders in old age leading to an increase in the incidence (and therefore the prevalence) of mental disorders. Also, life 
expectancy after the onset of mental disorders in old age may increase due to greater availability of appropriate healthcare services and treatment leading to an increase in the prevalence of such disorders. This is supported by positive correlations between per capita GDP and the proportion of GDP spent on health and per capita expenditure on health. This will lead to a gradual transition from a high prevalence-low socioeconomic status stage to a high prevalence-high socioeconomic status stage.

In socioeconomically more developed societies the prevalence of mental disorders in old age may progressively decline over many years due to advances in medical care. These societies are likely to have more readily available healthcare facilities and treatments for mental disorder in old age; this is supported by positive correlations between per capita GDP and the proportion of GDP spent on health and per capita expenditure on health. Increased availability of appropriate healthcare facilities and treatments may facilitate improved treatment of mental disorders and may lead to a reduction in the prevalence. Moreover, public health initiatives to enhance protective factors and reduce risk factors for mental disorders in old age are more likely to be available in socioeconomically more developed societies and may lead to a reduction in their incidence (and therefore their prevalence). Ultimately, this will lead to a gradual transition from a high prevalence-high socioeconomic society stage to a low prevalence-high socioeconomic society stage.

This epidemiological transition model is similar to a version of the model that has already been proposed for the epidemiological transition of dementia (Suh and Shah, 2001), but the current model could be applied empirically to all age-related disorders and elderly suicide rates.

\section{References}

Chandra, V. et al. (2001). Incidence of Alzheimer's disease in a rural community in the Indo-US study. Neurology, 57, 985-989.

Lindesay, J. (1981). Suicide in the elderly. International fournal of Geriatric Psychiatry, 6, 355-361.

McIntosh, J. L. (1984) Components of the decline in elderly suicides: suicide in young old and old old by race and sex. Death Education, 8, 113-124.

Seiden, R. H. (1981) Mellowing with age: factors affecting the non-white suicide rate. International fournal of Aging and Human Development, 13, 265-284.

Suh, G. H. and Shah, A. K. (2001). A review of the epidemiological transition in dementia: cross-national comparisons of indices related to Alzheimer's disease and vascular dementia. Acta Psychiatrica Scandinavica, 104, 4-11.

AJIT SHAH

Institute of Philosophy, Diversity and Mental Health, Centre for Ethnicity and Health, University of Central Lancashire, Preston, U.K. 